\title{
Optimal Signal Discrimination in a Low Signal-to-Noise Ratio Environment
}

Thiago Ciodaro ${ }^{1}$, on behalf of the ATLAS Tile calorimeter group ${ }^{1}$ Federal University of Rio de Janeiro

\section{1 - The ATLAS Detector}

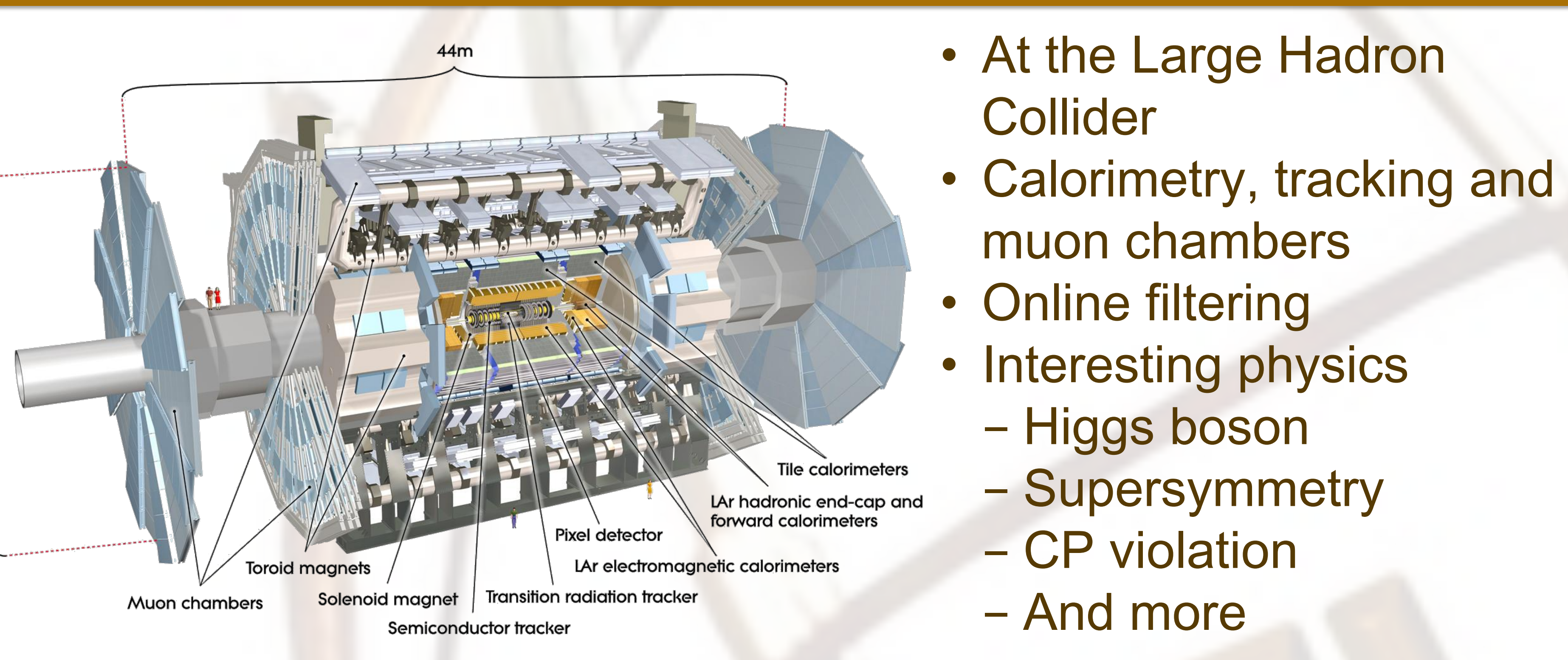

1.1 - Trigger System

- Three cascaded levels - Increasing complexity - Tight time constraints

- Level 1 trigger (L1)

- Implementation in-hardware

- Low granularity

- Calorimetry and muon spectrometer

\section{4 - Signal Discrimination}

- Identify summed Tile muon signals at the Tile muon receiver

- Constraint: simplicity for online implementation

- Matched filter (MF) based discriminators

- Based on the hypothesis test

$$
\Lambda(\mathbf{r}) \triangleq \frac{p d f_{\mathbf{r} \mid H_{1}}(\mathbf{r})}{p d f_{\mathbf{r} \mid H_{0}}(\mathbf{r})} \stackrel{\stackrel{H_{1}}{\gtrless} \gamma}{\stackrel{H_{0}}{\gtrless}} \quad \begin{array}{lll}
H_{0}: & r[k]=n[k] \\
H_{1}: & r[k]=n[k]+s[k] \longrightarrow \text { noise }
\end{array} \text { muon }
$$

- Testbeam data: signals from muon beams impinging Tile at different $\eta$ (incident polar angle)

- Muon receiver PSpice simulation (muon and noise signals)

- Digitization: 11 samples, 8-bits
- Data split into development and test sets $(50-50 \%)$

- Development: design the discriminators

- Test: evaluate their performance

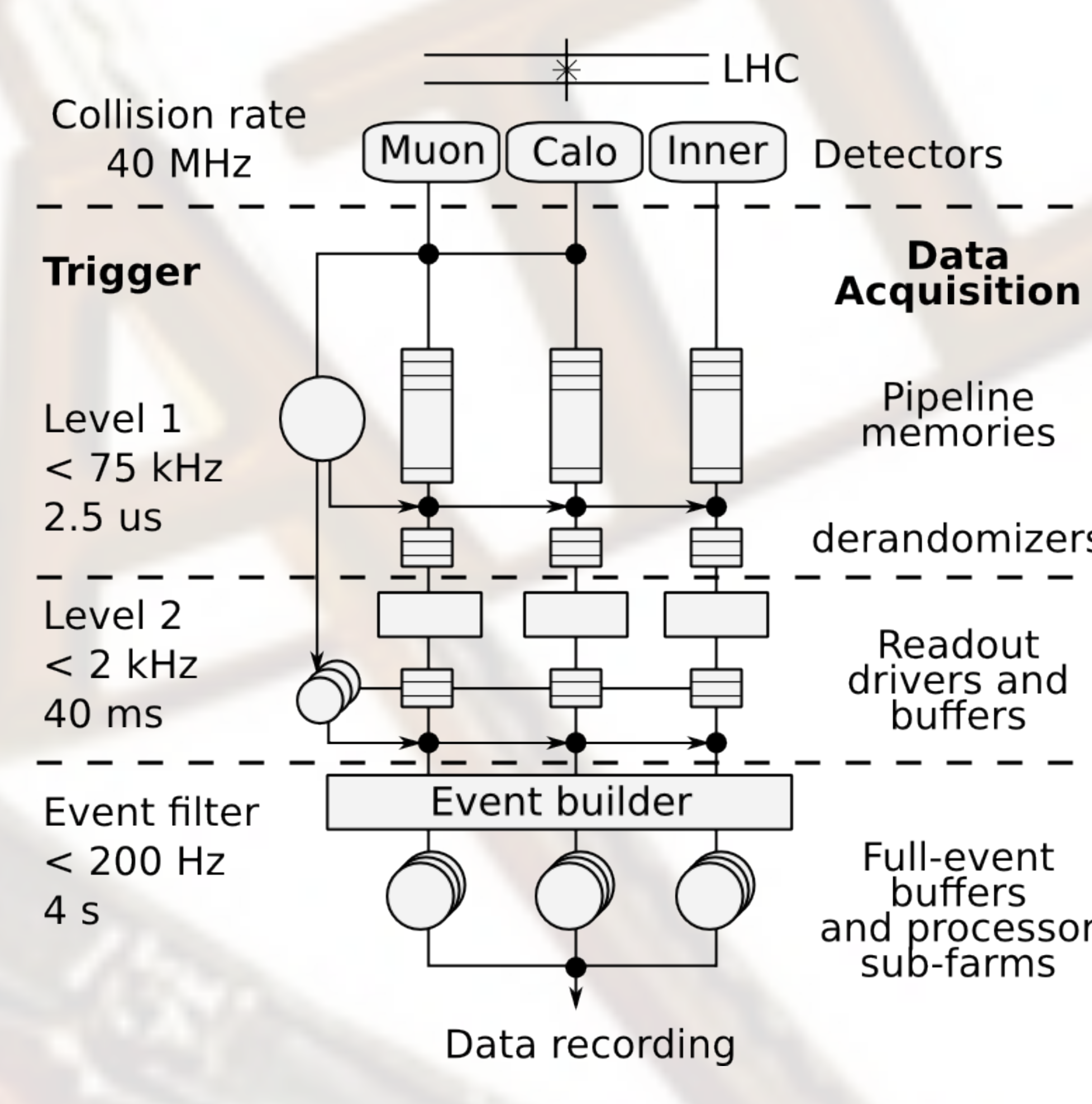

\section{2 - The Tile Calorimeter}

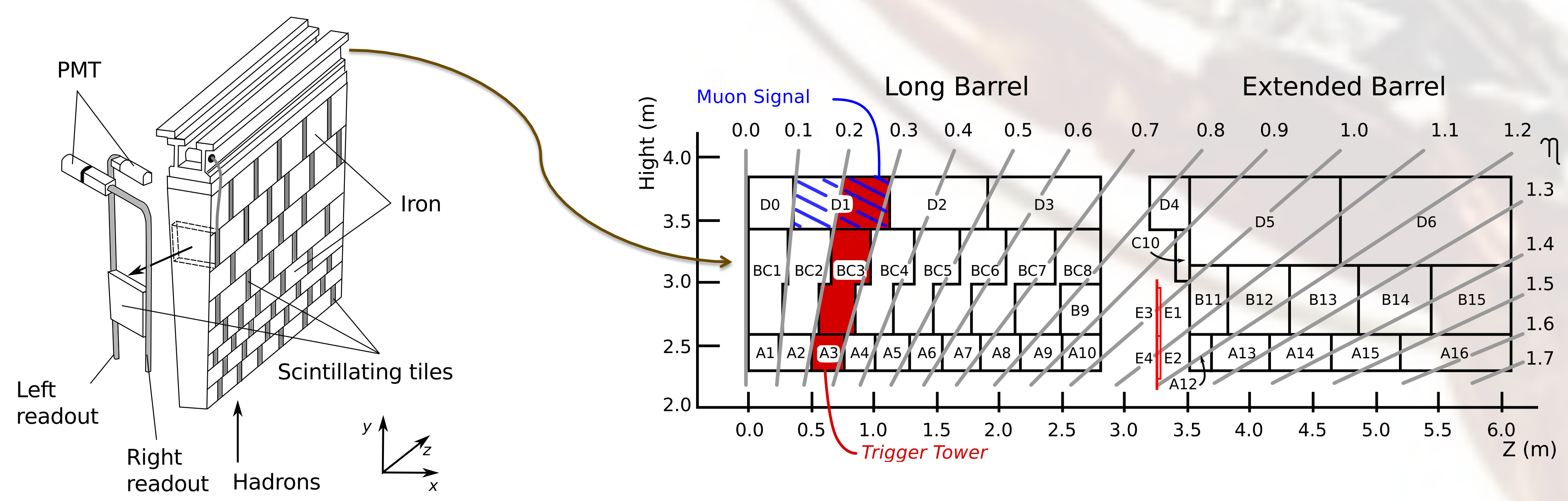

- Central hadronic calorimeter

- Energy measurements

- Iron as a structure and particle absorber, scintillating tiles as active medium

- Tiles grouped together into cells - Every cell read out by two independent fiber bundles and electronics

Muon: rear sampling (D cells) readouts (available at the L1 interface)

\section{1 - Matched filter: simplified approach}

- Muon: represented by the signal from its mean energy $(\mathrm{m}[\mathrm{k}])$

- Noise: zero-mean white Gaussian (w[k])

- Not white: whitening stage (W), based on the noise covariance matrix
$\begin{array}{ll}H_{0}: & r[k]=w[k] \\ H_{1}: & r[k]=m[k]+w[k]\end{array} \quad \Lambda(\mathbf{r})=\sum_{k=1}^{11} r[k] m[k]$
$[E, D]=\operatorname{eig}(\operatorname{cov}(w))$
$\mathbf{W}=D^{-1 / 2} E^{T}$

\section{1 - Matched filter: Gaussian signal approach}

- Muon: Gaussian (principal component analysis - PCA)

- Noise: zero-mean white Gaussian (w[k])

- Whitening stage as in the simplified approach

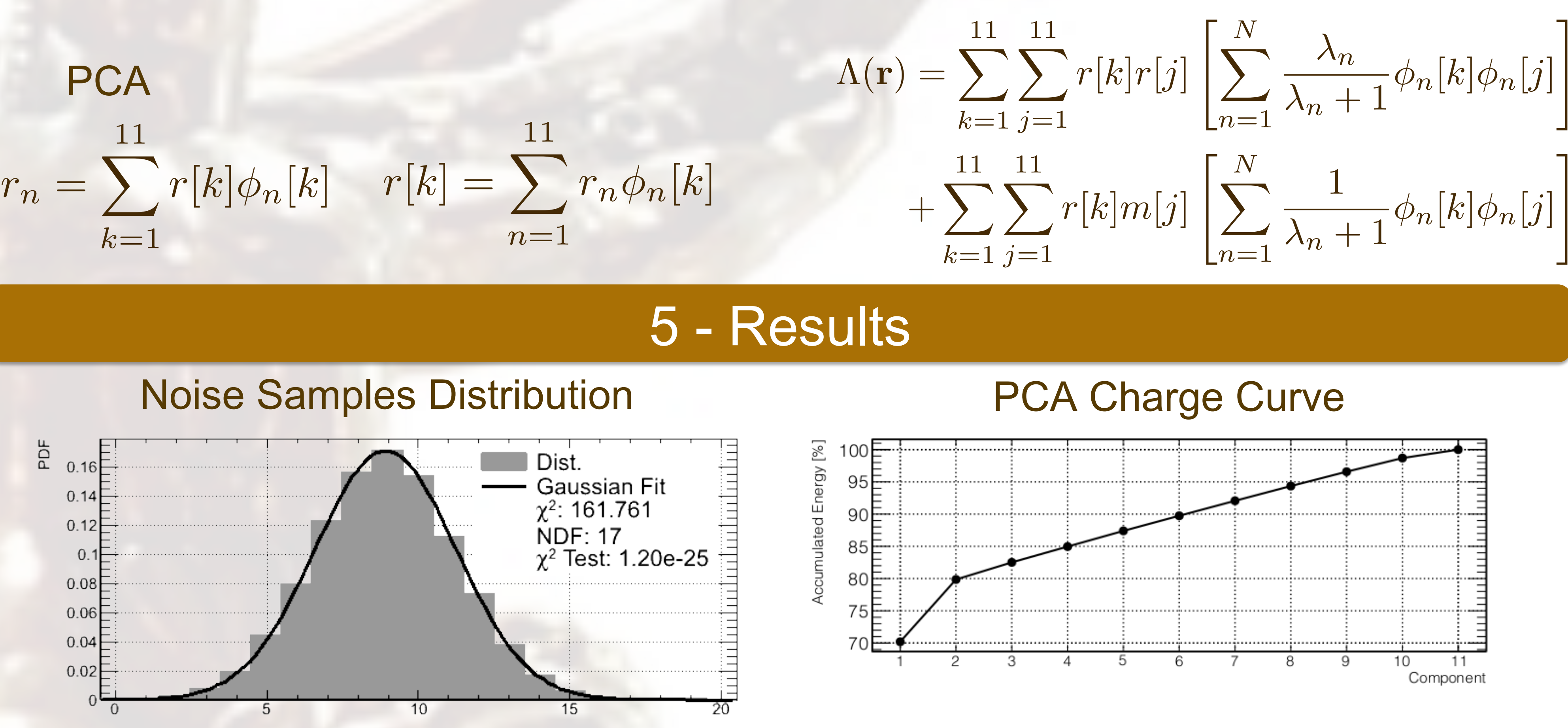

Chi-square test rejects Gaussian model • One PC holds $\sim 70 \%$ of the total muon

- The Jensen-Shannon test between the

summed signal information

\section{noise distribution and a random} generated Gaussian, with same mean and variance, confirms similarity

- Performance

- Increases for large energy deposition from muons

- Gaussian approach slightly better than the simple approach

- Gaussian with a single component ( $70 \%$ of the total information) also achieves good results

- Both matched filter approaches much better than a threshold discriminator over the digitized samples

- Same behavior seen for muons in all $n$

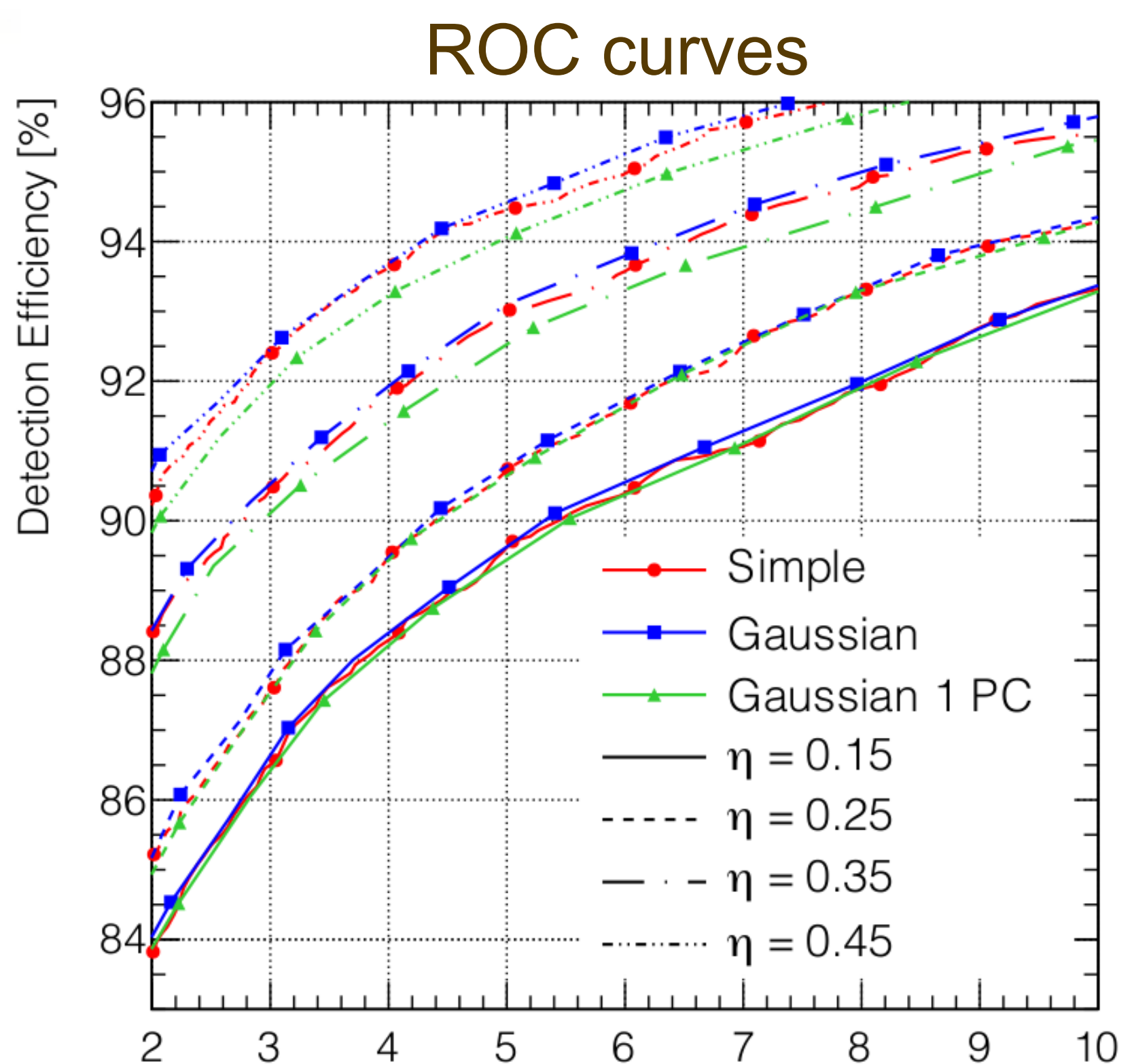

\begin{tabular}{|c|c|c|c|c|}
\hline Muon Detection (\%) & Threshold & MF Simple & MF Gaussian & MF Gaussian 1 PC \\
\hline$\eta=0.15$ & 80.1 & 89.5 & 89.5 & 89.5 \\
\hline$\eta=0.25$ & 81.5 & 90.9 & 90.9 & 90.9 \\
\hline$\eta=0.35$ & 82.4 & 93.0 & 93.1 & 92.5 \\
\hline$\eta=0.45$ & 83.0 & 94.5 & 94.5 & 94.0 \\
\hline
\end{tabular}

6 - Conclusions

- Discriminators based on matched filters achieve better results

- The simplified approach is preferred for online application 